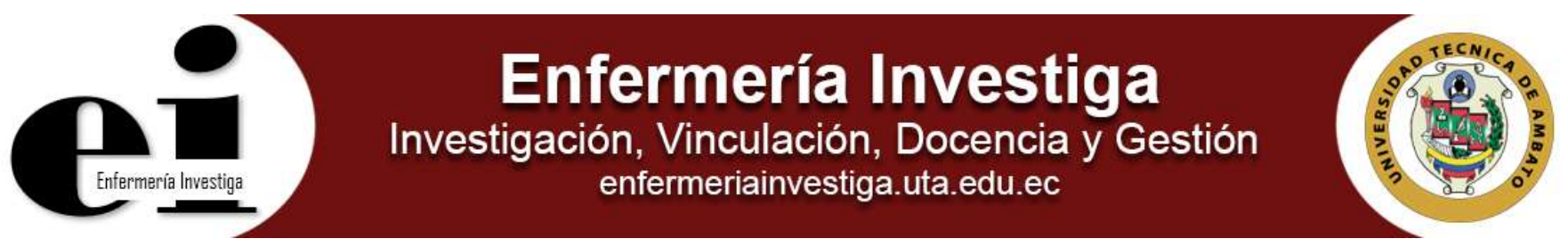

Artículo de revisión

\title{
Variaciones del perfil lipídico en pacientes pediátricos con desnutrición
}

Variations of the lipid profile in pediatric patients with malnutrition

\author{
Gerardo Fernando Fernández Soto ${ }^{1}$, Jean Carlos Quintero Nieto², Elsa Verónica Troya Ortiz ${ }^{3}$, Carolina Arráiz de \\ Fernández ${ }^{4}$, Tania Beatriz Romero-Adrian ${ }^{5}$
}

\begin{abstract}
${ }^{1}$ Médico Pediatra, Magister en Inmunología, PhD en Ciencias Médicas, Profesor de la Carrera de Estimulación Temprana, Facultad Ciencias de la Salud, Universidad Técnica de Ambato, Ambato, Ecuador.

${ }^{2}$ Facultad de Medicina, División de Estudios para Graduados, Programa de Especialización en Pediatría, Universidad del Zulia, Maracaibo, Venezuela.

${ }^{3}$ Magister en Educación Especial, Coordinadora Docente de la Carrera de Estimulación Temprana, Facultad Ciencias de la Salud, Universidad Técnica de Ambato, Ambato, Ecuador.

${ }^{4}$ Médica Pediatra-Neumóloga, Profesora de la Carrera de Laboratorio Clínico, Facultad Ciencias de la Salud, Universidad Técnica de Ambato, Ambato, Ecuador.

${ }^{5}$ Médica Parasitóloga, Magister en Inmunología, PhD en Ciencias Médicas, Profesora Titular y Coordinadora de la Maestría de Inmunología, Universidad del Zulia, Maracaibo, Venezuela.
\end{abstract}

Fernández SGF, Quintero NJC, Troya OEV, Arráiz deFC, Romero-Adrián TB. Variaciones del perfil lipídico en pacientes pediátricos con desnutrición. Enferm Inv (Ambato). 2017; 2(2):72-76.

2477-9172 / 2550-6692 Derechos Reservados @ 2017 Universidad Técnica de Ambato, Carrera de Enfermería. Este es un artículo de acceso abierto distribuido bajo los términos de la Licencia Creative Commons, que permite uso ilimitado, distribución y reproducción en cualquier medio, siempre que la obra original es debidamente citada.

\section{Historia:}

Recibido: 31 mayo 2017

Revisado: 02 junio 2017

Aceptado: 13 junio 2017

Palabras Claves:

Dislipidemias; lipoproteínas: perfil lipídico; desnutrición

Keywords: Dyslipidemias; lipoproteins; lipid profile; malnutrition

\begin{abstract}
Resumen
La desnutrición infantil se registra como uno de los principales problemas de salud pública de América Latina; pues es una de las mayores causas de mortalidad y morbilidad evitable en los niños y niñas de Latinoamérica. Adicional al problema de la alta prevalencia de la desnutrición infantil, son motivo de gran preocupación las consecuencias que la desnutrición deja en los niños que la sufren; ya que los niños con desnutrición en los primeros años de vida tienen mayor riesgo de sufrir enfermedades crónicas en la vida adulta, tales como enfermedad cardiovascular, accidente cerebrovascular y diabetes mellitus 2, donde la ateroesclerosis es el evento etiopatológico que enlaza la desnutrición y la enfermedad crónica. Uno de los factores implicados en la etiología de la ateroesclerosis en los niños que han padecido desnutrición, es la alteración en el metabolismo de los lípidos, propia de la enfermedad y que se evidencia por la modificación en la distribución relativa de los lípidos plasmáticos. Las investigaciones actuales demuestran que niños con desnutrición aguda presentaban un aumento tanto de los triglicéridos como del colesterol de las lipoproteínas de muy baja densidad con disminución del colesterol de las lipoproteínas de alta densidad.
\end{abstract}

\begin{abstract}
Child malnutrition is one of the main public health problems in Latin America; As it is one of the major causes of preventable mortality and morbidity in children in Latin America; In addition to the problem of high prevalence of child malnutrition, the consequences of malnutrition on children suffering from malnutrition are a matter of great concern; Since children with malnutrition in the first years of life are at higher risk for chronic diseases in adult life, such as cardiovascular disease, stroke and diabetes mellitus 2, where atherosclerosis is the etiopatological event that links malnutrition and disease chronicle. One of the factors implicated in the etiology of atherosclerosis in children who have suffered from malnutrition is the alteration in lipid metabolism, characteristic of the disease and evidenced by the change in the relative distribution of plasma lipids. Current research shows that children with acute malnutrition had an increase in both triglycerides and very low-density lipoprotein cholesterol with a decrease in high-density lipoprotein cholesterol.
\end{abstract}

Autor de correspondencia:

Gerardo Fernando Fernández Soto. Profesor de la Carrera de Estimulación Temprana, Facultad Ciencias de la Salud, Universidad Técnica de Ambato, Ambato, Ecuador. E-mail: gfernandez@uta.edu.ec 


\section{Introducción}

El estado nutricional está determinado por la ingestión, biodisponibilidad, utilización y reserva de nutrientes, que favorece el crecimiento y desarrollo adecuado de un individuo ${ }^{1}$. El estado nutricional del ser humano puede ser evaluado mediante diferentes indicadores antropométricos. Dentro de las medidas antropométricas más usadas se incluyen: peso, talla, circunferencia craneana, pliegues cutáneos, perímetros y circunferencias longitudinales y anchuras de segmentos corporales; a partir de ellas se puede describir una gran cantidad de índices ${ }^{1}$. Estas medidas antropométricas a su vez se correlacionan con edad y entre sí, y se comparan con los estándares vigentes para hacer la calificación y el diagnóstico nutricional. La calificación nutricional se realiza en base a la comparación de los indicadores peso para la edad $(\mathrm{P} / \mathrm{E})$, talla para la edad $(T / E)$ y peso para talla $(P / T)$, usando como referencia el patrón de la Organización Mundial de la Salud (OMS) ${ }^{2}$.

Entre los indicadores descritos, tiene mayor importancia en el menor de 1 año el indicador P/E y en el niño entre un año y los cinco años 11 meses 29 días el indicador P/T. La medición del peso para la edad, talla para la edad y el peso para la talla, constituyen los parámetros más fieles para evaluar crecimiento y estado nutritivo en el niño ${ }^{2}$. Los indicadores utilizados tienen la siguiente significancia:

- $\quad$ Peso para la edad (P/E): Es un buen indicador de déficit nutricional durante el primer año de vida, pero no permite diferenciar a niños constitucionalmente pequeños, por lo que su uso como parámetro único no es recomendable ${ }^{2}$. Refleja desnutrición actual.

- Talla para la edad (T/E): Diagnóstica desnutrición crónica. Es el indicador que se modifica a más largo plazo. El crecimiento lineal continuo es el mejor indicador de dieta adecuada y de estado nutricional a largo plazo, es un buen índice de cronicidad $^{2}$.

- $\quad$ Peso para la talla (P/T): Es un buen indicador de estado nutricional actual $y$ no requiere un conocimiento preciso de la edad. Es útil para el diagnóstico, tanto de desnutrición como de sobrepeso y obesidad. Se utiliza de dos a 10 años, pero no identifica a los niños desnutridos crónicos adaptados $^{2}$.

- Circunferencia Braquial para la edad: Efectivo en el diagnóstico de malnutrición por déficit. Su disminución implica perdida de grasa, musculo y ambos tejidos.

- Circunferencia Cefálica para la edad: Importante en menores de tres años. En desnutrición proteicocalórica esta disminuida, su aumento es signo de recuperación nutricional.

\section{Desarrollo}

La desnutrición infantil se define como el peso bajo para la talla (Peso/Talla), según la OMS dos desviaciones estándar (DE) por debajo de la mediana del peso esperado para la estatura $(P / T<-2 D E)$. De acuerdo con la intensidad se clasifica en: leve, moderada y en grave o severa. Sin embargo, es importante aclarar que, desde el punto de vista clínico la desnutrición grave se clasifica en tres categorías: 1) Marasmo con la presencia de emaciación severa 2) Kwashiorkor caracterizado por la presencia de edema de tipo nutricional y 3) mixta, en la que coexiste la emaciación severa con el edema de tipo nutricional ${ }^{3,4}$.

La desnutrición infantil se registra como uno de los principales problemas de salud pública y bienestar social de América Latina; pues es una de las mayores causas de mortalidad y morbilidad evitable en los niños de Latinoamérica; que además se encuentra relacionada por los deficientes determinantes sociales, económicos y políticas de salud de la gran mayoría de países de la región ${ }^{5}$. La desnutrición infantil sigue representando uno de los mayores desafíos en los países en vías de desarrollo ${ }^{5}$, es el resultado de carencias nutricionales graves y prolongadas de nutrientes para su desarrollo físico y mental; sin embargo existen otras causas subyacentes como son los factores socio-ambientales, la pobreza, falta de acceso a alimentos, falta de atención sanitaria, malas condiciones de vivienda, prácticas deficientes de cuidado y alimentación ${ }^{4}$.

Un porcentaje importante de los menores afectados por esta patología corresponden a edad preescolar y escolar, predominando el déficit nutricional en el sexo masculino, en niños procedentes de área metropolitana y la mayor parte pertenecen a la clase obrera, donde el jefe del hogar es desempleado y habitan en hogares con condiciones sanitarias deficientes y viviendas de mala calidad $^{6}$. El consumo de calorías disminuye consistentemente al descender en la escala de estratificación social de Graffar, evidenciándose significativa reducción de nutrientes entre estratos III, IV y V. Las deficiencias de crecimiento que ocurren en situaciones de pobreza involucran complejas interacciones entre carencias nutricionales, enfermedades y factores que tienen que ver con pautas y posibilidades en el cuidado de los niños, existiendo una relación directa entre el estrato social y el estado nutricional de estos niños ${ }^{7}$.

En Ecuador la desnutrición crónica afecta mayormente a los niños indígenas por sobre el promedio nacional, mientras que los afroecuatorianos se encuentran en mejores condiciones que este promedio. La desnutrición crónica se encuentra en mayores porcentajes en los indígenas residentes en la Región Sierra. En el área rural, los indígenas tienen un porcentaje mucho mayor de desnutrición crónica; mientras que los afroecuatorianos tienen similares porcentajes en las áreas urbana y rural, prevalece la desnutrición crónica en aquellos niños cuyos padres y madres indígenas poseen niveles de instrucción primaria y mayormente en aquellos que no tienen nivel de instrucción ${ }^{8}$.

Adicional al problema de alta prevalencia de la desnutrición infantil, son motivo de gran preocupación las consecuencias que la desnutrición deja en los niños que la sufren; Barker postuló que los niños con desnutrición en los primeros años de vida tienen mayor riesgo de sufrir enfermedades crónicas en la vida adulta, tales como enfermedad cardiovascular, accidente cerebrovascular y diabetes mellitus 2 , donde la ateroesclerosis es el evento etiopatológico que enlaza la desnutrición y la enfermedad crónica ${ }^{4}$.

Uno de los factores implicados en la etiología de la ateroesclerosis en los niños que han padecido desnutrición, es la alteración en el metabolismo de los lípidos, propia de la enfermedad y que se evidencia por la modificación en la distribución relativa de los lípidos plasmáticos. Las investigaciones actuales demuestran que niños con desnutrición aguda presentaban un aumento tanto de los triglicéridos (TG) como del colesterol de las lipoproteínas de muy baja densidad (C-VLDL), con disminución del colesterol de las lipoproteínas de alta densidad (C-HDL).

Hoy se sabe que la aterosclerosis es un proceso patológico que se instala silencioso desde la niñez; en consecuencia, los niños desnutridos con alteraciones de los lípidos circulantes y sometidos a tratamientos de recuperación nutricional, que propician crecimiento compensatorio, tienen 
un mayor riesgo aterogénico y más alta probabilidad de sufrir enfermedades crónicas asociadas a síndrome metabólico en años posteriores ${ }^{4}$.

Muchos trabajos han reportado que mientras mayor es el grado de la desnutrición, mayor es la concentración de TG y de colesterol de las C-VLDL en el plasma. Por el contrario, las concentraciones de colesterol total, C-LDL y C-HDL en plasma disminuyen a medida que aumenta la gravedad de la desnutrición ${ }^{9}$. Las dislipidemias o hiperlipidemias en niños y adolescentes son un grupo de trastornos que se caracterizan por una producción excesiva o aclaramiento disminuido de lipoproteínas séricas. Pueden ser primarias, de origen genético o hereditario, o secundarias asociadas a diferentes enfermedades ${ }^{12}$.

De acuerdo a Calzada y colaboradores, la dislipidemia se define por un colesterol total $\geq 200 \mathrm{mg} / \mathrm{dl}$, colesterol unido a lipoproteínas de baja densidad (C-LDL) $\geq 130 \mathrm{mg} / \mathrm{dL}$; triglicéridos $\geq 100 \mathrm{mg} / \mathrm{dl}$ y colesterol unido a lipoproteínas de alta densidad (C-HDL) $\leq 40 \mathrm{mg} / \mathrm{dl}^{13}$. La Clasificación de Cifras de Lípidos en Niños y Adolescentes de dos a 19 Años, según las recomendaciones de la American Heart Association (AHA), la Asociación Americana de Pediatría (AAP), aparecen en la tabla 1.

La enfermedad cardiovascular causada por la arterioesclerosis es una de las principales causas de muerte en el mundo, y está íntimamente relacionada con la dislipidemia. Está demostrado que la ateroesclerosis coronaria se desarrolla a lo largo de varios años con un probable inicio en la infancia. Hay evidencia que los elementos precursores de la ateroesclerosis y la producción de placas en la capa intima de las arterias, pueden iniciar desde la niñez, para desarrollarse plenamente en la tercera o cuarta décadas de la vida, aunque también pueden verse en la adolescencia. Esto ocurre como consecuencia de las alteraciones que sufre la pared vascular por procesos inflamatorios e inmunológicos, además de la influencia de la dislipidemia y otros factores metabólicos. Está claramente establecido que la presencia de factores de riesgo cardiovascular como la obesidad y la dislipidemia, están directamente relacionados con la formación de la placa ateromatosa ${ }^{10}$.

Estudios realizados como el de Bogalusa y el Young Finns Study, demostraron la relación que existe entre los niveles de colesterol en la edad pediátrica y en la edad adulta; evidencian que los niveles elevados de colesterol total y las C-LDL tienden a mantenerse en un mismo percentil a lo largo del tiempo en el mismo individuo. De aquí surge la preocupación de detectar y tratar precozmente a niños con hipercolesterolemia, dado que si se modifican los factores de riesgo en las fases iníciales de la ateroesclerosis, se pudiera experimentar regresión de la placa pre-ateromatosa o al menos detener el proceso ${ }^{10}$.

Existen múltiples evidencias científicas que apoyan la asociación de los niveles adversos en los patrones de lípidos y lipoproteínas en el desarrollo de enfermedades cardiovasculares más tarde en la vida. En el estudio de Muscatine, donde analizaron los factores de riesgo de enfermedades cardiovasculares en niños y adultos en edad escolar, la ecografía carotídea en adultos indicó que el grosor de la íntima-media de la carótida (CIMT, siglas en inglés) se asoció positivamente con los niveles de colesterol total medidos en la infancia. Resultados similares fueron establecidos en el Bogalusa Heart Study, en el que se utilizaron las C-LDL de la infancia para predecir aumento CIMT en la edad adulta ${ }^{11}$.

En otro estudio finlandés, las asociaciones entre la exposición a los factores de riesgo en la adolescencia, incluyendo C-LDL, predijo CIMT en la edad adulta, independiente de los niveles de factor de riesgo para adultos; por lo tanto hay más pruebas de que los factores de riesgo comienza en la infancia y se asocian con los mismos factores de riesgo de enfermedad cardiovascular que están bien establecidos en los adultos ${ }^{10,11}$.

Considerando que el proceso de ateroesclerosis se inicia en etapas tempranas de la vida, una detección oportuna de dislipidemia mejora su pronóstico y previene complicaciones en la vida adulta. Un diagnóstico temprano se puede realizar mediante un interrogatorio de antecedentes familiares, hábitos alimentarios y un recordatorio de dieta de uno a tres días, el registro de peso, talla e índice de masa corporal y la medición de colesterol, triglicéridos y perfil de lipoproteínas. La intervención nutricional y médica temprana puede garantizar una mejor calidad de vida en niños, adolescentes. Las principales medidas de prevención primaria de enfermedad cardiovascular ateroesclerótica, que se inician en la infancia están relacionadas con una dieta balanceada en la que se busca mantener un perfil de lípidos adecuado ${ }^{12}$.

\section{El Tamizaje de dislipidemias en la infancia}

La pesquisa de dislipidemias en pediatría se realizaba teniendo en cuenta el riesgo. Se buscaba identificar y estudiar aquellos niños con antecedentes familiares de enfermedad cerebrovascular temprana, dislipidemia, antecedentes familiares de diabetes, obesidad, hipertensión y tabaquismo, entre otros; o aquellos niños cuyos antecedentes familiares fueran desconocidos.

Tabla 1: Clasificación de Cifras de Lípidos en Niños y Adolescentes de dos a 19 años.

\begin{tabular}{|c|c|c|c|}
\hline Parámetros & $\begin{array}{c}\text { Aceptable } \\
(\mathrm{mg} / \mathrm{dl}) \\
(<\mathrm{p} 75)\end{array}$ & $\begin{array}{l}\text { Límite o riesgo moderado } \\
\text { (mg/dl) } \\
(\text { p 75-94) }\end{array}$ & $\begin{array}{c}\text { Inaceptable } \\
\text { (mg/dl) } \\
(\geq p 95)\end{array}$ \\
\hline $\begin{array}{l}\text { Triglicéridos } \\
\qquad \quad 0-9 \text { años } \\
\bullet \quad 10-19 \text { años } \\
\text { Colesterol total } \\
\text { C-LDL }\end{array}$ & $\begin{array}{l}<75 \\
<90 \\
<170 \\
<110\end{array}$ & $\begin{array}{c}75-99 \\
90-129 \\
170-199 \\
110-129\end{array}$ & $\begin{array}{l}>100 \\
>130 \\
\geq 200 \\
\geq 130\end{array}$ \\
\hline Parámetro & $\begin{array}{c}\text { Aceptable } \\
(\mathrm{mg} / \mathrm{dl}) \\
(>\mathrm{p} 25)\end{array}$ & $\begin{array}{l}\text { Límite } \\
(\mathrm{mg} / \mathrm{dl}) \\
\text { (p 10-25) }\end{array}$ & $\begin{array}{l}\text { Disminuido } \\
(\mathrm{mg} / \mathrm{dl}) \\
(<\mathrm{p} 10)\end{array}$ \\
\hline C-HDL & $>45$ & $40-45$ & $<40$ \\
\hline
\end{tabular}

C: colesterol; C-HDL: colesterol lipoproteína de alta densidad; C-LDL: colesterol lipoproteína de baja densidad. 
Este abordaje tradicional resulta insuficiente ya que muestra una eficacia menor del $50 \%$, debido a que existe un subdiagnóstico en adultos que desconocen su perfil lipídico y su historia familiar. Esto limita la posibilidad diagnóstica en los niños, con la consiguiente pérdida de la oportunidad de instaurar un tratamiento en caso de ser necesario. Existen formas genéticas de dislipidemias que aumentan el riesgo cardiovascular tempranamente, son asintomáticas y requieren de un tratamiento adecuado, lo que remarca la necesidad del diagnóstico precoz ${ }^{10}$. En 2011, se conformó un panel de expertos de la AAP y el Instituto Nacional del Corazón, Sangre y Pulmón de Estados Unidos (NHLBI, siglas en inglés); los que crearon las guías para la prevención del riesgo cardiovascular en pediatría, en las que se propuso un tamizaje universal de dislipidemias ${ }^{12}$.

El tamizaje universal tiene como objetivo pesquisar dislipidemias de moderadas a graves en niños aparentemente sanos y sin antecedentes familiares conocidos que deberían ser tratadas. El beneficio secundario es que, a partir de los niños, podrán diagnosticarse sus padres, quienes tienen riesgo inmediato de enfermedad cardiovascular. Aquellos niños con riesgo de enfermedad cerebrovascular elevado, ya sea por antecedentes familiares conocidos o por padecer patologías con reconocido impacto en la salud cardiovascular, deben ser estudiados a partir de los dos años de vida o cuando se diagnostica su enfermedad de base ${ }^{13-15}$.

\section{Dislipidemias y factores genéticos}

Las concentraciones plasmáticas de lípidos están determinadas en una elevada proporción por factores genéticos. En sus formas más extremas se manifiestan como hiperlipemias familiares. El hipercolesterolemia familiar (HF) es una enfermedad genética monogénica, caracterizada por un defecto en la captación celular de las lipoproteínas plasmáticas, predominantemente $\mathrm{LDL}^{16,17}$. Entre estas destacan por su frecuencia y su elevado riesgo cardiovascular, la hiperlipemia familiar combinada (HFC), con una prevalencia de al menos $1: 100$ y la HF con 1:250300 . Se calcula que ambas pueden ser la causa de hasta un $40 \%$ de la enfermedad coronaria prematura. La HFC se caracteriza por altas concentraciones de C-LDL, TG o ambos y se asocia con frecuencia a diabetes tipo 2, hipertensión arterial y sobrepeso central. Por lo tanto, conocerla es muy importante para que el médico pueda realizar un diagnóstico y tratamiento precoces ${ }^{18}$.

\section{Conclusiones}

La enfermedad cardiovascular causada por la arterioesclerosis es una de las principales causas de muerte en el mundo, y está íntimamente relacionada con la dislipidemia. Está demostrado que la ateroesclerosis coronaria se desarrolla a lo largo de varios años con un probable inicio en la infancia. Los niños desnutridos con alteraciones de los lípidos circulantes y sometidos a tratamientos de recuperación nutricional, que propician crecimiento compensatorio, tienen un mayor riesgo aterogénico y más alta probabilidad de sufrir enfermedades crónicas asociadas a síndrome metabólico en años posteriores, es decir mientras mayor es el grado de la desnutrición, mayor es la concentración de TG y de C-VLDL en el plasma. Por lo tanto es fundamental brindar asesoramiento nutricional a los padres de familia, cuyos hijos presentan desnutrición, realizando charlas educativas sobre la alimentación balanceada y la utilización oportuna de la pirámide de los alimentos, también es importante incorporar dentro del protocolo de atención de los niños desnutridos, la determinación del perfil lipídico, recordando elevada asociación entre dislipidemia y desnutrición.

\section{Referencias}

1. Obando Borja ML. Factores determinantes de estado nutricional (educación materna, nivel socioeconómico e ingesta dietética), de niños y niñas de 6 y 7 años de la Escuela Ernesto Noboa y Caamaño en el cantón Cayambe, provincia de Pichincha [en línea] [B.S. thesis]. Quito, 2014; 2014 [citado 11 de enero de 2017]. Disponible en: http://repositorio.usfq.edu.ec/handle/23000/3566

2. Nelson Campos Villalobos, Ada Vergara Doyhamboure, Carolina González Bruna. Norma para el Manejo Ambulatorio de la Malnutrición por Déficit y Exceso en el Niño/a Menor de 6 años. Ministerio de Salud de Chile (2007)

3. Kac G, Alvear G, L J. Epidemiología de la desnutrición en Latinoamérica: situación actual. Nutrición Hospitalaria [en línea]. 2010 [citado 13 de febrero de 2017];25:50-6. Disponible en: http://scielo.isciii.es/scielo.php?script=sci abstract\&pid=S0212$16112010000900008 \&$ Ing=es\&nrm=iso\&tlng=pt

4. Rodríguez CMV, Cano JMO. Dislipidemia en niños con desnutrición aguda. Turbo, Colombia. Perspectivas en Nutrición Humana [en línea] 2013 [citado 10 de marzo de 2017];14(2). Disponible en: http://aprendeenlinea.udea.edu.co/revistas/index.php/nutricion/article/ view/16486

5. Acevedo E, Sanabria MC, Delgadillo JL, Durán CC. Kwashiorkor y marasmo-kwashiorkor en niños hospitalizados. Pediatría (Asunción) [en línea]. 2013 [citado 21 de febrero de 2017];31(1):16-22. Disponible en: http://revista.spp.org.py/index.php/ped/article/view/178

6. Hernández $M$, Salinas PJ. Relación entre estado nutricional y características socioeconómicas en pre-escolares, Mérida, Venezuela. MedULA [en línea]. 1992 [citado 13 de marzo de 2017];1(3):110-4. Disponible en: http://erevistas.saber.ula.ve/index.php/medula/article/view/5566

7. Torres-Cárdenas $M$, Pérez BM, Landaeta-Jiménez $M$, VásquezRamírez M. Consumo de alimentos y estado nutricional según estrato socioeconómico en una población infantil de Caracas. Arch venez pueric pediatr [en línea]. 2011 [citado 12 de enero de 2017];74(2):2-9. Disponible en: http://www.redalyc.org/pdf/3679/367937042002.pdf

8. Instituto Nacional de Estadísticas y Censos. La desnutricion en la poblacion indigena y afroecuatoriana. Análisis Sociodemográfico. [en línea]. Quito, Ecuador; 2008 [citado 11 de mayo de 2017] p. 28 Disponible en: http://www.ecuadorencifras.gob.ec/documentos/webinec/Bibliotecas/Estudios/Estudios_Socio-

demograficos/La_Desnutricion_en_la_Poblacion_Indigena_y_Afroec uat.pdf

9. Dini E, Arenas O. Pruebas de laboratorio en niños con desnutrición aguda moderada. An Venez Nutr [en línea]. 2002 [citado 15 de febrero de 2017];15(2):67-75. Disponible en http://www.cania.org.ve/PDF/investigacion/Trabajos_2002_salud_2.p df

10. Shrestha R, Copenhaver M. Long-Term Effects of Childhood Risk Factors on Cardiovascular Health During Adulthood. Clinical medicine reviews in vascular health [en línea]. 2015[citado 11 de mayo de 2017];7:1-5. Disponible http://www.ncbi.nlm.nih.gov/pmc/articles/PMC4547483/

11. Merino de Méndez G. Manejo de las dislipidemias en niños y adolescentes. Archivos venezolanos de puericultura y pediatría [en línea]. 2007 [citado 21 de febrero de 2017];70(4). Disponible en: http://www.redalyc.org/html/3679/367935539006/

12. Heller-Rouassant S. Dislipidemias en niños y adolescentes: diagnóstico y prevención. Boletín médico del Hospital Infantil de México [en línea]. 2006 [citado 15 de marzo de 2017];63(3):158-161. Disponible en: http://scielo.unam.mx/scielo.php?pid=S1665$11462006000300002 \&$ script=sci_arttext\&tlng=es

13. Araujo M. Consenso sobre manejo de las dislipidemias en pediatría. Archivos Argentinos de Pediatría [en línea]. [citado 22 de enero de 2017]; 113(2):177-186 Disponible en:

http://www.sap.org.ar/docs/publicaciones/archivosarg/2015/v113n2a2 3.pdf

14. Barja S, Baeza C, Hodgson MI, others. Diagnóstico y tratamiento de las dislipidemias en niños y adolescentes: Recomendaciones de la Rama de Nutrición de la Sociedad Chilena de Pediatría. Revista chilena de pediatría [en línea]. 2014 [citado 16 de enero de 2017];85(3):367-377. 410620140003000148 s.php?pid=S0370-

15. Nielsen TRH, Lausten-Thomsen U, Fonvig CE, Bøjsøe C, Pedersen L, Bratholm PS, et al. Dyslipidemia and reference values for fasting plasma lipid concentrations in Danish/North-European White children and adolescents. BMC Pediatrics [en línea]. diciembre de 2017 [citado 10 de marzo de 2017];17(1). Disponible en: http://bmcpediatr.biomedcentral.com/articles/10.1186/s12887-0170868-y

16. Alberico L. Catapano, lan Graham, Guy De Backer, Olov Wiklund. 2016/EAS Guidelines for the Management of Dyslipidaemias. Eur Heart J (2016) 37 (39): 2999-3058. 
17. Civeira F, Plana N. Treatment of Heterozygous Familial Hypercholesterolemia in Children and Adolescents: An Unsolved Problem. Revista Española de Cardiología (English Edition) [en línea]. 2017[citado 23 de febrero de 2017];70(6):423-4. Disponible en: http://www.sciencedirect.com/science/article/pii/S1885585717300701
18. Catapano Leopoldo, Sánchez PL , Álvarez-Sala L, Barrios V, Castro A, Galve E, García L, Mata P. Comments on the 2016 ESC/EAS Guidelines for the Management of Dyslipidemias. Rev Esp Cardiol. 2017 [citado 23 de febrero de 2017];70(2):115. Disponible en: http://linkinghub.elsevier.com/retrieve/pii/S1885585717300026

Enferm Inv. (Ambato) 2(2):72-76 